\title{
A Standard Form for the Dynamics of General Manipulation Systems
}

\author{
Antonio Bicchi ${ }^{\dagger}$ \\ Domenico Prattichizzo * \\ †Centro "E.Piaggio" \& Dipartimento di Sistemi Elettrici ed Automazione, Università di Pisa - Pisa, Italy \\ ‡Artificial Intelligence Laboratory, Massachusetts Instistute of Technology - Cambridge, Massachusetts
}

\begin{abstract}
In this paper we consider the structural properties of the dynamics of robotic manipulation systems of a rather general class, including multiple cooperating, possibly whole-arm limbs, interacting with a manipulated object by means of contacts. A geometric approach to the analysis of the linearized dynamics of such systems is presented, which provides much insight in some of their intrinsic characteristics in the light of classical system-theoretic concepts such as controllability, observability, and canonical forms.
\end{abstract}

\section{Introduction}

A growing interest is developing in the robotics community towards manipulation systems with features such as multi-limb coordination, whole-arm manipulation, and/or underactuated joints. Instances of such mechanisms are e.g. robotic hands, cooperating robot arms, or legged vehicles. By "whole-limb" manipulation we design a style of manipulation where all the links in the limb (including proximal ones) are exploited to interact with the manipulated object (Salisbury, [1987]). A peculiarity of whole-limb systems is their defect of d.o.f. in their operational space.

The purpose of this paper is to analyze the dynamics and the system-theoretic structural properties of such class of systems. Although dynamics may not play a dominant role in the control of slow cooperating manipulative tasks, only a full dynamical model can explain and clarify the structural properties of complex manipulation systems. Thus, dynamic manipulation has been considered to investigate grasp stability (e.g. by Nakamura et al. [1989], Montana [1991], Trinkle [1992], and Howard and Kumar [1994]); and cooperative manipulability ([Chiacchio et al., 1991]). As we consider more general manipulation systems, other structural properties enter the picture, related in particular to restrictions to controllability/observability entailed by kinematic defectivity. This point represents the focus of the present paper.

\section{Dynamic Model}

A manipulation system is a constrained mechanical system, whose dynamical description can be de-

*on leave from Dipartimento di Sistemi Elettrici ed Automazione, Univerisità di Pisa

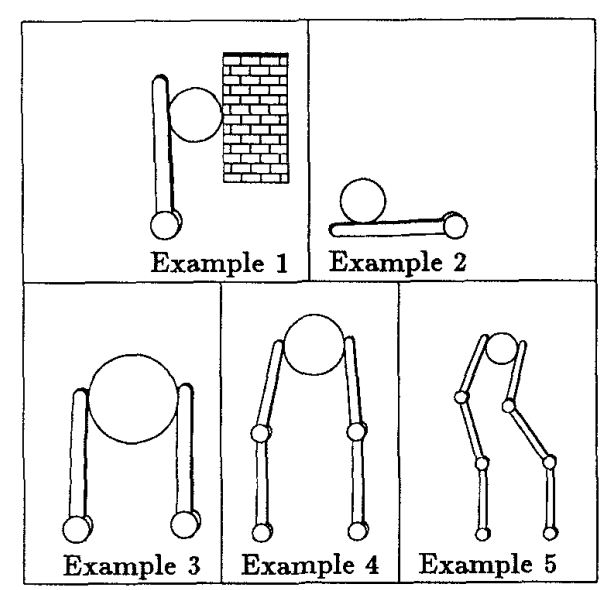

Table 1: Five simple examples of robotic manipulators.

rived using Euler-Lagrange's equations along with constraint equations. As already discussed elsewhere ([Bicchi and Prattichizzo, 1993]), a rigid-body model of general manipulation systems (GMS) is not satisfactory because of two reasons. First, for kinematically defective systems, dynamics cannot be completely specified, and unmodelled elastic energy can be stored in non-independent (hyperstatic) constraints. Second, closed-loop force control in rigid-body models entails algebraic loops, and unmodeled dynamics would show up in applications. We therefore introduce a model of visco-elastic interactions at the contacts between the links of the limbs and the object. For simplicity, we assume that bodies are linearly elastic. Also, we postulate that contact points do not change by rolling. The latter assumptions is motivated by the lack of a tractable model of rolling and compliant contacts ([Johnson, 1985]).

According to the above discussion, the model of a manipulation system can be written as follows. Let $\mathbf{q} \in \mathbb{R}^{q}$ denote the vector of joint positions, and let $\mathbf{u} \in \mathbb{R}^{d}$ be the vector locally describing the position 
and orientation of a frame attached to the object. Correspondingly, let $\tau \in \mathbb{R}^{q}$ be the vector of joint actuator forces and torques, and $\mathbf{w} \in \mathbb{R}^{d}$ the vector of forces and torques resultant from actions applied directly at the object. The dynamics of the limbs (considered as a whole and henceforth indicated by the term "hand"), and that of the object can be written as

$$
\begin{aligned}
& \mathbf{M}_{h}(\mathbf{q}) \ddot{\mathbf{q}}+\mathbf{Q}_{h}(\mathbf{q}, \dot{\mathbf{q}})+\mathbf{J}^{T}(\mathbf{q}, \mathbf{u}) \mathbf{t}=\boldsymbol{\tau} \\
& \mathbf{M}_{o}(\mathbf{u}) \ddot{\mathbf{u}}+\mathbf{Q}_{o}(\mathbf{u}, \dot{\mathbf{u}})-\mathbf{G}(\mathbf{u}, \mathbf{q}) \mathbf{t}=\mathbf{w}
\end{aligned}
$$

respectively, where the $\mathbf{M}_{i}(\cdot)(i=h, o)$ are inertia matrices, the $\mathbf{Q}_{i}(\cdot, \cdot)$ terms include velocity-dependent and gravity forces, $\mathbf{J}$ is the aggregated (pseudo)Jacobian matrix for the hand, and $\mathbf{G}$ is the so-called "grasp" matrix. Finally, $t$ is a $t$-dimensional vector of all components of contact forces between the links and the object, which, according to the lumped springdamper model we assume, can be written as

$$
\mathbf{t}=\mathbf{K} \boldsymbol{\xi}(\mathbf{q}, \mathbf{u})+\mathbf{B}\left(\mathbf{J} \dot{\mathbf{q}}-\mathbf{G}^{T} \dot{\mathbf{u}}\right) .
$$

Here, $\mathbf{K}$ and $\mathbf{B}$ are aggregated matrices of contact stiffness and damping, respectively, and $\xi$ is a suitable displacement function applied to the positions and orientations of surface (Gauss) frames at the contact points.

\subsection{Linearization}

For the analysis of most of the structural properties of WAM systems, model (1)-(2) is still intractable. Henceforth, then, we will deal with the linearized dynamic model

$$
\dot{\mathbf{x}}=\mathbf{A x}+\mathbf{B}_{\tau} \tau+\mathbf{B}_{w} \mathbf{w}
$$

where the state vector $\mathbf{x} \in \mathbb{R}^{2(q+d)}$, inputs $\tau^{\prime} \in \mathbb{R}^{q}$, and disturbances $\mathbf{w}^{\prime} \in \mathbb{R}^{d}$ are defined as the departures from a reference equilibrium configuration $\mathbf{x}_{o}=\left[\begin{array}{llll}\mathbf{q}_{o}^{T} & \mathbf{u}_{o}^{T} & \mathbf{0}^{T} & 0^{T}\end{array}\right]^{T}$ at which contact forces are $\mathbf{t}\left(\mathbf{x}_{o}\right)=\mathbf{t}_{o}$. The dynamics matrix $\mathbf{A}$, joint torque input matrix $\mathbf{B}_{\tau}$, and external wrench disturbance matrix $\mathbf{B}_{w}$ have the form

$$
\mathbf{A}=\left[\begin{array}{cc}
\mathbf{0} & \mathbf{I} \\
\mathbf{L}_{k} & \mathbf{L}_{b}
\end{array}\right] ; \mathbf{B}_{\tau}=\left[\begin{array}{c}
\mathbf{0} \\
\mathbf{0} \\
\mathbf{M}_{h}^{-1} \\
\mathbf{0}
\end{array}\right] ; \mathbf{B}_{w}=\left[\begin{array}{c}
\mathbf{0} \\
\mathbf{0} \\
\mathbf{0} \\
\mathbf{M}_{o}^{-1}
\end{array}\right]
$$

In the general case, blocks $\mathbf{L}_{k}$ and $\mathbf{L}_{b}$ still have rather involved expressions in terms of the system's kinematic and material properties, and depend on the intensity of forces at equilibrium. To the purpose of obtaining clearly intelligible results relating structural properties of manipulation systems to their more intrinsic parameters, the linearized model is considered under the further assumptions that: i) gravity terms are negligible in $\mathbf{Q}_{h}$ and $\mathbf{Q}_{o}$; ii) stiffness and damping are isotropic at each contact, i.e. there exists positive constants $\kappa_{i}$ and $\beta_{i}$ such that, in a local frame, $\mathbf{K}_{i}=\kappa_{i} \mathbf{I}$ and $\mathbf{B}_{i}=\beta_{i} \mathbf{I}$; and iii) contact forces at the reference equilibrium are small, so that terms $\frac{\theta \mathbf{J}^{\mathbf{T}} \mathbf{t}_{\mathrm{o}}}{\partial \mathbf{q}}$, $\frac{{ }_{\theta} \mathbf{G t}_{o}}{\partial \mathbf{u}}$ are negligible. Under these conditions, we have

$$
\mathbf{L}_{k}=-\mathbf{M}^{-1} \mathbf{P}_{k} ; \quad \mathbf{L}_{b}=-\mathbf{M}^{-1} \mathbf{P}_{b},
$$

where $\mathbf{M}=\operatorname{diag}\left(\mathbf{M}_{h}, \mathbf{M}_{o}\right)$, and

$\mathbf{P}_{k}=\left[\begin{array}{c}\mathbf{J}^{T} \\ -\mathbf{G}\end{array}\right] \mathbf{K}\left[\begin{array}{ll}\mathbf{J} & -\mathbf{G}^{T}\end{array}\right]=\left[\begin{array}{cc}\mathbf{J}^{T} \mathbf{K J} & -\mathbf{J}^{T} \mathbf{K G}^{T} \\ -\mathbf{G K J} & \mathbf{G} \mathbf{K G}^{T}\end{array}\right]$,

and $\mathbf{P}_{b}$ has the same form as $\mathbf{P}_{k}$ where $\mathbf{K}$ is replaced by $\mathbf{B}$.

\section{Pointwise Controllability}

The subspace of states that are pointwisecontrollable from joint torques for the linearized system (4), denoted by $\left\langle\mathbf{A} \mid \mathbf{B}_{\tau}\right\rangle$, can be simply analyzed if contact damping is neglected, i.e. if $\mathbf{B}=\mathbf{0}$. In such a case, putting

$$
\Lambda=-\mathbf{K}\left(\mathbf{J} \mathbf{M}_{h}^{-1} \mathbf{J}^{T}+\mathbf{G}^{T} \mathbf{M}_{o}^{-1} \mathbf{G}\right),
$$

we have that the columns of the controllability matrix can be written as

$$
\begin{aligned}
\mathbf{A}^{2 k} \mathbf{B}_{\tau} & =\left(\mathbf{M}_{h}^{-1} \mathbf{J}^{T} \Lambda^{k-1} \mathbf{K}\left[\mathbf{0} 0-\mathbf{J M}_{h}{ }^{-1} \mathbf{G}^{T} \mathbf{M}_{o}^{-1}\right]\right)^{T} ; \\
\mathbf{A}^{2 k+1} \mathbf{B}_{\tau} & =\left(\mathbf{M}_{h}^{-1} \mathbf{J}^{T} \Lambda^{k-1} \mathbf{K}\left[-\mathbf{J M}_{h}{ }^{-1} \mathbf{G}^{T} \mathbf{M}_{\circ}^{-1} \mathbf{0} 0\right]\right)^{T} .
\end{aligned}
$$

After some calculations, one obtains

$$
\langle\mathbf{A}| \mathbf{B}_{\tau}>=\left\{\mathbf{x}\left|\Delta \mathbf{u}, \dot{\mathbf{u}} \in<\mathbf{M}_{o}^{-1} \mathbf{G K G} \mathbf{G}^{T}\right| \mathbf{M}_{o}^{-1} \mathbf{G K J}>\right\}
$$

The following cases may be encountered:

1. If the Jacobian $\mathbf{J}$ and the grasp matrix $\mathbf{G}$ are full row rank (f.r.r.), the system is completely controllable. Such is the case for examples 4 and 5 in table 1 .

2. If $\operatorname{ker}\left(\mathbf{G}^{T}\right) \neq \emptyset$, the system is called indeterminate. If furthermore $\mathbf{J}$ is f.r.r., the controllable subspace is

$$
<\mathbf{A} \mid \mathbf{B}_{\tau}>=\left\{\mathbf{x} \mid \Delta \mathbf{u}, \dot{\mathbf{u}} \in \quad\left(\mathbf{M}_{o}^{-1} \mathbf{G}\right)\right\} .
$$

Note that only object displacements and velocities belonging to range $\left(\mathbf{M}_{o}^{-1} \mathbf{G}\right)$ are reachable. In particular (since $\mathbf{M}_{o}$ is p.d.), the indeterminate subspace

$$
\mathcal{X}_{\mathbf{i}}=\left\{\mathbf{x} \mid \Delta \mathbf{q}=\dot{\mathbf{q}}=\mathbf{0}, \Delta \mathbf{u}, \dot{\mathbf{u}} \in \operatorname{ker}\left(\mathbf{G}^{T}\right)\right\},
$$

is not reachable.

3. If $\operatorname{ker}\left(\mathbf{J}^{T}\right) \neq \emptyset$, the system is called defective. Defective systems (with $G$ f.r.r.) may or may not loose complete controllability, depending on the particular case considered. Controllability of defective systems is generic (in the sense of algebraic geometry, cf. e.g. Wonham [1979]). For the device in example 3 of table 1 controllability of vertical and rotational movements of the object is lost due to the particular symmetry of inertia, stiffness and damping parameters that were assumed in the introduction. The same holds for the example 1 of table 1 . Table 3 reports graphical illustrations of the uncontrollable modes in 
these cases. Note that the loss of controllability in indeterminate systems is generic.

4. If neither $\mathbf{J}$ nor $\mathbf{G}$ are f.r.r., the system is defective and indeterminate. This is the case of example 2 of table 1.

Observing that the indeterminate subspace $\mathcal{X}_{i}$ is A-invariant; and applying a state space transformation $\mathbf{T}_{1}=\left[\mathbf{T}_{\tau}\left|\mathbf{T}_{\boldsymbol{i}}\right| \mathbf{T}_{d}\right]$ whereof $\mathbf{T}_{\tau}$ is a basis matrix ${ }^{1}$ (b.m.) of $\left\langle\mathbf{A} \mid \mathbf{B}_{\tau}\right\rangle, \mathbf{T}_{i}$ is a b.m. of $\mathcal{X}_{i}$, and $\mathbf{T}_{d}$ is a complementary basis matrix ${ }^{2}$ (c.b.m.) of $<\mathbf{A} \mid \mathbf{B}_{\tau}>\oplus \mathcal{X}_{i}$ to the state space $\mathbb{R}^{2(q+d)}$, a standard controllability form for GMS is obtained as

$$
\mathbf{T}_{1}^{-1} \mathbf{A} \mathbf{T}_{1}=\left[\begin{array}{c|c|c}
{ }^{\tau} \mathbf{A} & \mathbf{0} & \star \\
\hline \mathbf{0} & { }^{i} \mathbf{A} & \star \\
\hline \mathbf{0} & \mathbf{0} & { }^{d} \mathbf{A}
\end{array}\right] ; \quad \mathbf{T}_{\mathbf{1}}^{-1} \mathbf{B}_{\tau}=\left[\begin{array}{c}
\bullet \\
\hline \mathbf{0} \\
\hline \mathbf{0}
\end{array}\right]
$$

where the symbol "•" is for non-zero blocks, while the symbol " $\star$ " represents blocks that may be zero or not depending on the particular choice of the complementary basis matrix. This form of the dynamics of a GMS points out that uncontrollable modes may appear because of two reasons. The modes associated with ${ }^{i} \mathbf{A}$ are the indeterminate modes of the system, and are strictly related to the existence of a nullspace of the transpose of the grasp matrix, in the sense that they correspond to motions left free by the grasp. Indeterminate modes are double integrators. The uncontrollable modes associated with ${ }^{d} \mathbf{A}$ are the defective modes of the system, since a necessary condition for their existence is that the hand Jacobian has not full row rank. This case occurs in WAM systems but also in conventional robots at their kinematic singularities (see table 2). Defective modes are periodic in the assumption that the damping matrix $\mathbf{B}$ is zero, and are damped by positive definite $\mathbf{B}$ 's.

In general, if damping is present at the contacts, the above standard form remains valid. However, the dimension of the pointwise-state controllable subspace is generically increased whenever the damping matrix $\mathbf{B}$ and the stiffness matrix $\mathbf{K}$ do not commute.

\section{Observability}

Being the goal of dextrous manipulation to control the position of the manipulated object through the contact forces with the fingers, it is natural to consider two possible outputs, namely the object position $\mathbf{u}$ and the contact force vector $\mathbf{t}$. In the linearized model under consideration, the corresponding output matrices are written respectively as

$$
\begin{gathered}
\mathbf{C}_{\mathbf{t}}=\left[\begin{array}{llll}
\mathbf{K J} & -\mathbf{K G}^{T} & \mathbf{B J} & -\mathbf{B G}^{T}
\end{array}\right] ; \\
\mathbf{C}_{\mathbf{u}}=\left[\begin{array}{llll}
0 & \mathbf{I} & 0 & 0
\end{array}\right] .
\end{gathered}
$$

\footnotetext{
${ }^{1} \mathbf{V}$ is called a basis matrix of a subspace $\mathcal{V}$ if it is f.c.r. and $\mathbf{V}=\boldsymbol{V}$.

${ }^{2} \mathbf{W}$ is called a complementary basis matrix of $\mathcal{V}$ to $\mathcal{W}$ if it is f.c.r. and $(\mathbf{W}) \oplus \mathcal{V}=\mathcal{W}$.
}

\subsection{Observability from Object Motions}

The rows of the observability matrix $\mathbf{O}_{\mathbf{u}}$ from the positions of the object are

$$
\begin{aligned}
& \mathbf{C}_{\mathbf{u}}(\mathbf{A})^{2 k}=\mathbf{M}_{o}^{-1} \mathbf{G} \Lambda^{k-1} \mathbf{K}\left[\mathbf{J}-\mathbf{G}^{T} \mathbf{0} 0\right] ;
\end{aligned}
$$

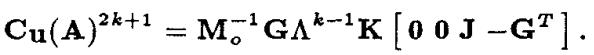

The subspace of states unobservable from $\mathbf{u}$ is

$$
\operatorname{ker}\left(\mathbf{O}_{\mathbf{u}}\right)=\left\{\mathbf{x} \mid \Delta \mathbf{q}, \dot{\mathbf{q}} \in \mathcal{V}_{h}, \Delta \mathbf{u}=\dot{\mathbf{u}}=\mathbf{0}\right\}
$$

where $\mathcal{V}_{h}$ is the largest subspace contained in $\operatorname{ker}(\mathbf{G K J})$ that is $\left(\mathbf{M}_{h}^{-1} \mathbf{J}^{T} \mathbf{K J}\right)$-invariant, i.e.

$$
\mathcal{V}_{h}=\bigcap_{i=1}^{q+d-1} \operatorname{ker}\left(\left[\mathbf{G K J} \mathbf{J}\left(\mathbf{M}_{h}^{-1} \mathbf{J}^{T} \mathbf{K J}\right)^{i-1}\right]\right)
$$

The following remarks apply here:

1. If $\mathbf{J}$ and $\mathbf{G}$ are f.c.r., the system is completely observable from object motions, as in example 2 of table 1 .

2 . If $\operatorname{ker}(\mathbf{J}) \neq \emptyset$, the system is called redundant. If furthermore $\mathbf{G}$ is f.c.r., the subspace unobservable from object motions is

$$
\mathcal{X}_{r}=\{\mathbf{x} \mid \Delta \mathbf{q}, \dot{\mathbf{q}} \in \operatorname{ker}(\mathbf{J}), \Delta \mathbf{u}=\dot{\mathbf{u}}=\mathbf{0}\}
$$

that is, it is comprised of redundant joint displacements and velocities. The existence of an unobservable subspace in redundant systems is generic.

3. If $\operatorname{ker}(\mathbf{G}) \neq \emptyset$, the system is called graspable (the name follows from the fact that contact forces in $\operatorname{ker}(\mathbf{G})$ are usually called internal (or grasping) forces, and play a fundamental role in resisting external disturbances with unilateral friction contact constraints). Graspable systems (assuming $\mathbf{J}$ is f.c.r.) may or may not loose complete observability, depending on the particular case considered (i.e., observability is generic for graspable, non-redundant systems). Elements of the unobservable subspace are joint displacements and rates that modify contact forces, but leave object dynamics unmodified. The elements of the corresponding subspace of contact forces, $\mathcal{F}_{h}=\mathbf{C}_{t} \operatorname{ker}\left(\mathbf{O}_{\mathbf{u}}\right)$, are called dynamically internal contact forces. The possibility of exerting internal forces without affecting the motions of the object is of great practical relevance to cases when the demand of accuracy of manipulation is highest, as for instance when the object of manipulation is a surgical tool. In the apparently similar systems of examples 1 and 3 in table 1 the possibility of exerting dynamically internal forces is illustrated. No dynamically internal force can be exerted in example 1 , being void the intersection between the column space of $\mathbf{J}$ and the nullspace of $\mathbf{G}$ (this is depicted in table 3 , first row). In example 3 , however, this intersection is not void and, due to the particular symmetry of kinematic and inertial parameters, a dynamically internal contact force can be exerted as illustrated in table 3 , second row.

4. If neither $\mathbf{J}$ nor $\mathbf{G}$ are f.c.r., the system is redun- 
dant and graspable (see e.g. example 5 in table 1 ). Notice that the subspace of redundant motions is mapped in null contact forces by $\mathbf{C}_{\mathbf{t}}$.

Observing that the subspace $\mathcal{X}_{r}$ is $\mathbf{A}$-invariant, a standard observability form can be obtained as

$$
\begin{aligned}
\mathbf{T}_{2}^{-1} \mathbf{A} \mathbf{T}_{2} & =\left[\begin{array}{c|c|c}
{ }^{u} \mathbf{A} & \mathbf{0} & \mathbf{0} \\
\hline \star & \mathbf{A} & \star \\
\hline \star & \mathbf{0} & { }^{h} \mathbf{A}
\end{array}\right] \\
\mathbf{C}_{\mathbf{u}} \mathbf{T}_{2} & =\left[\begin{array}{l|l|l}
\mathbf{0} & \mathbf{0} & \mathbf{0}
\end{array}\right]
\end{aligned}
$$

where $\mathbf{T}_{\mathbf{2}}=\left[\mathbf{T}_{u}\left|\mathbf{T}_{r}\right| \mathbf{T}_{h}\right]$, and $\mathbf{T}_{r}$ is a b.m. of $\mathcal{X}_{r}, \mathbf{T}_{h}$ is a c.b.m. of $\mathcal{X}_{\boldsymbol{r}}$ to $\operatorname{ker}\left(\mathbf{O}_{\mathbf{u}}\right)$, and $\mathbf{T}_{u}$ is a c.b.m. of $\operatorname{ker}\left(\mathbf{O}_{\mathbf{u}}\right)$ to the state space $\mathbb{R}^{2(q+d)}$.

Modes that are unobservable from object positions may arise because of two reasons. "Redundant" modes associated with ${ }^{r} \mathbf{A}$ are present whenever the Jacobian matrix has a nullspace (as in example 5 of table 1). The redundant modes are double integrators, but can be arbitrarily relocated by feedback of joint variables only. The modes associated with ${ }^{h} \mathbf{A}$ are called " $\mathrm{dy}-$ namically internal" modes of the system, because of their relation with dynamically internal forces. The observability standard form is maintained for non-zero damping $(\mathbf{B} \neq \mathbf{0})$. However, the dimension of the dynamically internal subspace is generically decreased if $\mathbf{B}$ and $\mathbf{K}$ do not commute.

\subsection{Observability from Contact forces}

The analysis of state observability from the contact forces provides further insight in the kinematics of robotic systems. The rows of the observability matrix $\mathbf{O}_{\mathbf{t}}$ from the output $\mathbf{t}$ can be written, for $\mathbf{B}=\mathbf{0}$, as

$$
\begin{aligned}
\mathbf{C}_{\mathbf{t}} \mathbf{A}^{2 k} & =\Lambda^{k-1} \mathbf{K}\left[\begin{array}{llll}
\mathbf{J} & -\mathbf{G}^{T} & \mathbf{0} & 0
\end{array}\right] ; \\
\mathbf{C}_{\mathbf{t}} \mathbf{A}^{2 k+1} & =\Lambda^{k-1} \mathbf{K}\left[\begin{array}{llll}
\mathbf{0} & \mathbf{0} & \mathbf{J} & -\mathbf{G}^{T}
\end{array}\right]
\end{aligned}
$$

The subspace of states unobservable from contact forces is therefore

$$
\operatorname{ker}\left(\mathbf{O}_{\mathbf{t}}\right)=\left\{\mathbf{x} \mid\left[\begin{array}{c}
\Delta \mathbf{q} \\
\Delta \mathbf{u}
\end{array}\right],\left[\begin{array}{c}
\dot{\mathbf{q}} \\
\dot{\mathbf{u}}
\end{array}\right] \in \operatorname{ker}\left(\left[\mathbf{J}-\mathbf{G}^{T}\right]\right)\right\}
$$

and corresponds to displacements and velocities that leave the virtual springs and dampers unsolicited, i.e., to the rigid-body kinematics of the system.

Rigid-body kinematics are of particular interest in the control of robotic manipulation systems, because the extent to which displacements from the reference equilibrium comply with the linearized model is much limited for motions that involve visco-elastic deformations of bodies. Rigid kinematics can be characterized in terms of a matrix $\Gamma$ whose columns form a basis for $\operatorname{ker}\left(\left[\mathbf{J}-\mathbf{G}^{T}\right]\right)$, and that can be written as

$$
\Gamma=\left[\begin{array}{ccc}
\Gamma_{r} & 0 & \Gamma_{q c} \\
0 & \Gamma_{i} & \Gamma_{u c}
\end{array}\right]
$$

where $\Gamma_{r}$ is a b.m. of $\operatorname{ker}(\mathbf{J}), \Gamma_{i}$ is a b.m. of $\operatorname{ker}\left(\mathbf{G}^{T}\right)$, and $\Gamma_{q c}$ and $\Gamma_{u c}$ are conformal partitions of a c.b.m. to $\operatorname{ker}\left(\left[\mathbf{J}-\mathbf{G}^{T}\right]\right)$ of $\operatorname{diag}(\operatorname{ker}(\mathbf{J}), \operatorname{ker}(\mathbf{G}))$. The analysis of the dimensions and the geometry of the subspaces spanned by the blocks of matrix $\Gamma$ is instrumental in describing fundamental kinematic characteristics of robotic manipulation systems, such as the mobility, connectivity, and manipulability of manipulation systems. For instance, the structure described in example 1 of table 1 has no possible rigid motion $(\Gamma=0)$, as motions of the object may only result from deformations of the compliant elements at the contacts. Bicchi, Melchiorri, and Balluchi [1994] derived a similar description of rigid-body kinematics from quasi-static considerations, and had a detailed discussion on mobility and manipulability properties.

Applying a state space transformation $\mathbf{T}_{\mathbf{3}}=$ $\left[\mathbf{T}_{t}\left|\mathbf{T}_{r}\right| \mathbf{T}_{i} \mid \mathbb{T}_{c}\right]$, where $\mathbf{T}_{c}$ is a c.b.m. of $\mathcal{X}_{r} \oplus \mathcal{X}_{i}$ to $\operatorname{ker}\left(\mathbf{O}_{\mathbf{t}}\right)$, in particular

$$
\mathbf{T}_{c}=\left[\begin{array}{cc}
\Gamma_{q c} & \mathbf{0} \\
\Gamma_{u c} & \mathbf{0} \\
\mathbf{0} & \Gamma_{q c} \\
\mathbf{0} & \Gamma_{u c}
\end{array}\right]
$$

and $\mathbf{T}_{t}$ is a c.b.m. of $\operatorname{ker}\left(\mathbf{O}_{\mathbf{t}}\right)$ to $\mathbb{R}^{2(q+d)}$, and observing that range $\left(\mathbf{T}_{c}\right)$ is $\mathbf{A}$-invariant, a standard observability form is obtained as

$$
\begin{aligned}
\mathbf{T}_{3}^{-1} \mathbf{A} \mathbf{T}_{3} & =\left[\begin{array}{c|c|c|c}
{ }^{t} \mathbf{A} & \mathbf{0} & \mathbf{0} & \mathbf{0} \\
\hline \star & { }^{\boldsymbol{}} \mathbf{A} & \mathbf{0} & \mathbf{0} \\
\hline \star & \mathbf{0} & { }^{i} \mathbf{A} & \mathbf{0} \\
\hline \star & \mathbf{0} & \mathbf{0} & { }^{\boldsymbol{c}} \mathbf{A}
\end{array}\right] ; \\
\mathbf{C}_{\mathbf{t}} \mathbf{T}_{\mathbf{3}} & =\left[\begin{array}{l|l|l|l}
\bullet & \mathbf{0} & \mathbf{0} & \mathbf{0}
\end{array}\right]
\end{aligned}
$$

\section{Standard Form for GMS}

The dynamic structure of a general manipulation system, analyzed from different viewpoints in the preceding sections, can be summarized by a single result to be discussed shortly. As a necessary preliminary, however, we briefly consider here the dual properties to pointwise controllability from joint torques and to observability from object positions that were discussed previously. Such duals are observability from the position of joints (i.e., with output matrix $\mathbf{C}_{\mathbf{q}}=\left[\begin{array}{llll}\mathbf{I} & \mathbf{0} & 0 & 0\end{array}\right]$ ), and controllability from the disturbance input matrix $\mathbf{B}_{\mathbf{w}}$, respectively. In fact, one has

$$
\operatorname{ker}\left(\mathbf{O}_{\mathbf{q}}\right)=\left\{\mathbf{x} \mid \Delta \mathbf{q}=\dot{\mathbf{q}}=\mathbf{0}, \Delta \mathbf{u}, \dot{\mathbf{u}} \in \mathcal{W}_{h}\right\},
$$

where

$$
\mathcal{W}_{h}=\bigcap_{i=1}^{q+d-1} \operatorname{ker}\left(\left[\mathbf{J}^{T} \mathbf{K G}^{T}\left(\mathbf{M}_{o}^{-1} \mathbf{G} \mathbf{K G}^{T}\right)^{i-1}\right]\right)
$$

and

$\left\langle\mathbf{A} \mid \mathbf{B}_{\mathbf{w}}\right\rangle=\left\{\mathbf{x}\left|\Delta \mathbf{q}, \dot{\mathbf{q}} \in<\mathbf{M}_{h}^{-1} \mathbf{J}^{T} \mathbf{K} \mathbf{J}\right| \mathbf{M}_{h}^{-1} \mathbf{J}^{T} \mathbf{K} \mathbf{G}^{T}>\right\}$.

The following theorem provides a rather interesting standard form of GMS's without indetermination $\left(\operatorname{ker}\left(\mathbf{G}^{T}\right)=\emptyset\right)$. 
Theorem 1 The linearized model (4) of a robotic manipulation system with $\operatorname{ker}\left(\mathbf{G}^{T}\right)=\emptyset$ admits a change of coordinates $\mathbf{T}$ such that the system matrices, in the new coordinates, have the form

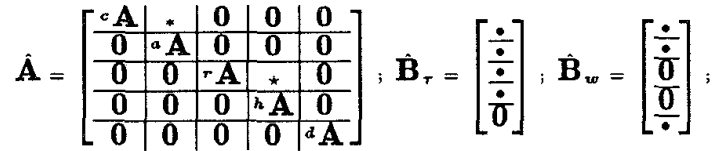

$$
\begin{aligned}
& \hat{\mathbf{C}}_{\mathbf{u}}=[\cdot|\cdot| \mathbf{0}|\mathbf{0}| \cdot] ; \\
& \hat{\mathbf{C}}_{\mathbf{q}}=[\cdot|\cdot| \cdot|\cdot| \mathbf{0}] \text {; } \\
& \mathbf{C}_{\mathbf{t}}=[0|\cdot| 0|\cdot| \cdot],
\end{aligned}
$$

where the symbol "*" represents a block that may be zero or not depending on the values of the dynamic and stiffness parameters $(*$ blocks are however generically non-zero).

The following lemmata involving previously defined subspaces and basis matrices are instrumental to the proof of theorem 1:

Lemma $1 \operatorname{ker}\left(\mathbf{O}_{\mathbf{u}}\right) \subseteq\left\langle\mathbf{A} \mid \mathbf{B}_{\tau}\right\rangle$ and $\operatorname{ker}\left(\mathbf{O}_{\mathbf{q}}\right) \subseteq$ $<\mathbf{A} \mid \mathbf{B}_{w}>$.

Proof. Directly from comparison of (9) and (6) and of (14) and (15).

Lemma 2 range $\left(\mathbf{T}_{c}\right) \subseteq\left\langle\mathbf{A} \mid \mathbf{B}_{\tau}\right\rangle$.

Proof. From comparison of (13) and (6), it appears that the thesis holds iff

$$
\operatorname{range}\left(\Gamma_{u c}\right) \subseteq<\mathbf{M}_{o}^{-1} \mathbf{G K G} \mathbf{T}^{T} \mid \mathbf{M}_{o}^{-1} \mathbf{G K J}>.
$$

$\begin{array}{lccr}\text { Observe first that } & \text { range }\left(\Gamma_{u c}\right) & \subseteq \\ \text { range }\left(\left(\mathbf{G K} \mathbf{K G}^{T}\right)^{-1} \mathbf{G K J}\right), & \text { or, } & \text { equiv- }\end{array}$ alently, that $\forall \alpha, \exists \beta: \Gamma_{u c} \alpha=\left(\mathbf{G K G}^{T}\right)^{-1} \mathbf{G K J} \beta$. From (12) in fact we get $\beta=\Gamma_{q c} \alpha$. The thesis follows by application of Cailey-Hamilton theorem to show that

$$
\begin{aligned}
& \operatorname{range}\left(\left(\mathbf{G K G} \mathbf{G}^{T}\right)^{-1} \mathbf{G K J}\right) \subseteq \\
& \left.\sum_{k=1}^{q+d-1} \operatorname{range}\left(\left(\mathbf{M}_{o}^{-1} \mathbf{G K G}\right)^{T}\right)^{k-1} \mathbf{M}_{o}^{-1} \mathbf{G K J}\right)
\end{aligned}
$$

Lemma 3 range $\left(\mathbf{T}_{c}\right) \cap\left(\mathcal{X}_{r} \oplus \operatorname{range}\left(\mathbf{T}_{h}\right)\right)=\emptyset$.

Proof. It follows from the definition of $\mathbf{T}_{h}$ and from comparison of (13) with (9).

$\operatorname{Lemma} 4<\mathbf{A} \mid \mathbf{B}_{\tau}>\oplus \operatorname{ker}\left(\mathbf{O}_{\mathbf{q}}\right)=\mathbb{R}^{2(q+d)}$.

Proof. We assume here that the representation of states is normalized so as to have homogeneous physical dimensions for all states, and to allow the definition of an internal product in the state space. Since such a normalization can always be obtained by means of a linear transformation of coordinates that is p.d., no loss of generality will ensue from this procedure of proof. Under these conditions, the following relationship involving orthogonal complements holds

$$
\begin{aligned}
\mathcal{W}_{h}^{\perp} & =\left(\bigcap_{i=1}^{q+d-1} \operatorname{ker}\left[\mathbf{J}^{T} \mathbf{K G}^{T}\left(\mathbf{M}_{o}^{-1} \mathbf{G} \mathbf{K G}^{T}\right)^{i-1}\right]\right)^{\perp} \\
& \left.=\mathbf{M}_{o} \sum_{k=1}^{q+d-1} \operatorname{range}\left(\mathbf{M}_{o}^{-1} \mathbf{G K G}\right)^{T}\right)^{k-1} \mathbf{M}_{o}^{-1} \mathbf{G K J} \\
& =\mathbf{M}_{o}<\mathbf{M}_{o}^{-1} \mathbf{G} \mathbf{K G}^{T} \mid \mathbf{M}_{o}^{-1} \mathbf{G K J}>.
\end{aligned}
$$

The thesis is proved by comparing (6) and (14) and considering that $\mathbf{M}$ is p.d..

$\operatorname{Lemma} \mathbf{5}<\mathbf{A} \mid \mathbf{B}_{w}>\oplus \operatorname{ker}\left(\mathbf{O}_{\mathbf{u}}\right)=\mathbb{R}^{2(q+d)}$.

Proof. Similar to proof of lemma 4.

Proof of Theorem 1. From lemmata 1, 2, and 3, a b.m. for the subspace of states controllable from joint

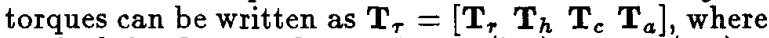
$\mathbf{T}_{a}$ is defined as a c.b.m. of range $\left(\mathbf{T}_{r}\right) \oplus \operatorname{range}\left(\mathbf{T}_{h}\right) \oplus$ range $\left(\mathbf{T}_{c}\right)$ to $\left\langle\mathbf{A} \mid \mathbf{B}_{\tau}\right\rangle$. Being $\mathcal{X}_{i}=\emptyset$, lemma 4 guarantees that $\mathbf{T}_{d}$ is a valid b.m. of $\operatorname{ker}\left(\mathbf{O}_{\mathbf{q}}\right)$ while lemma 5 guarantees that $\mathbf{T}_{h}$ is a valid c.b.m. of $\mathcal{X}_{\tau} \oplus<\mathbf{A} \mid \mathbf{B}_{w}>$ to $\mathbb{R}^{2(q+d)}$. The thesis follows from comparison of the canonical (Kalman) decompositions of systems $\left(A, B_{\tau}, \mathbf{C}_{\mathbf{u}}\right)$ and $\left(A, B_{\mathbf{w}}, \mathbf{C}_{\mathbf{q}}\right)$, that can be obtained by reordering the blocks of the following change of coordinates that is chosen as new basis,

$$
\mathbf{T}=\left[\begin{array}{lllll}
\mathbf{T}_{c} & \mathbf{T}_{a} & \mathbf{T}_{r} & \mathbf{T}_{h} & \mathbf{T}_{d}
\end{array}\right]
$$

Remark 1. One useful aspect of the standard form of theorem 1 consists in the synthetic representation of information relating to the structural properties of various subsystems. It can be shown in fact that it is always possible to choose $\mathbf{T}_{a}$ and $\mathbf{T}_{h}$ such that the $\star$ terms vanish in the standard form of theorem 1. Therefore, as it can be easily recovered from application of the Popov-Belevitch-Hautus test, the lack of one of the five properties considered (controllability from joint torques and from disturbances, observability from object positions, from joint positions, and from contact forces) for a particular subsystem is indicated by the presence of a zero block in the corresponding position of the input or output matrices.

\section{Discussion}

In this paper, the structure of dynamic systems for manipulation of objects has been investigated from the viewpoint of linear systems theory. Although robotic systems are highly nonlinear in nature, the simplicity of results achievable by linearization appeared to be important at this rather early stage of investigation of complex manipulation systems. For instance, it was possible to show that the dynamics and structural 
properties of whole-arm manipulation systems have nonnegligible differences from those of non-defective systems. Moreover, it is well known that some of the results on the linearized system imply analogous local properties for the full system. As one example of the possible practical relevance of the results of this paper, we should like to point out the definition and characterization of "dynamically internal" contact forces, that might be an important tool in designing and controlling devices for high-precision, surgicaltype ("steady-hand") robotic applications.

\section{Acknowledgements}

The research reported in this paper has been partially supported by the C.N.R. - P.F.R. under contracts 93.01079.PF67 and 93.01047.PF67.

\section{References}

Bicchi, A., and Prattichizzo, D.: "New Issues in the Kineto-Statics, Dynamics, and Control of Whole-Hand Manipulation", in Robotics, Mechatronics and Manufacturing Systems, T. Takamori and K. Tsuchiya (Editors), Elsevier Science Publishers B.V., North Holland, 1993.

Bicchi, A., Melchiorri, C., and Balluchi, D.:"On the Mobility and Manipulability of General Multiple Limb Robots", IEEE Trans. Robotics and Automation. In press. 1993.

Chiacchio, P., S. Chiaverini, L. Sciavicco, B. Siciliano: "Task space dynamic analysis of multiarm system configurations", International Journal of Robotics Research vol. 10, no. 6, pp. 708-715, 1991

Cole, A.A., Hsu, P., and Sastry, S.S.: "Dynamic Control of Sliding by Robot Hands for Regrasping", IEEE Trans. on Robotics and Automation, vol. 8, no. 1, pp. 42-52. February 1992.

Howard, W. S., and Kumar, V.: "Stability of Planar Grasps", Proc. IEEE Int. Conf. on Robotics and Automation, pp. 2822-2827, 1994.

Johnson, K.L. "Contact Mechanics", Cambridge University Press, 1985.

Montana, D.J.: “The Condition for Contact Grasp Stability", Proc. IEEE Cond. Robotics and Automation, pp. 412-417. 1991.

Nakamura, Y., Nagai, K., and Yoshikawa, T.: "Dynamics and Stability in Coordination of Multiple Robotic Systems", Int. Jour. of Robotic Research, vol.8, no.2, pp. 44-61. April 1989.

Salisbury, J. K.: "Whole-Arm Manipulation", Proc. of the 4-th International Symposium of Robotics Research, Santa Cruz, CA. MIT Press, 1987.

Trinkle, J.C.: "On the Stability and Instantaneous Velocity of Grasped Frictionless Objects", IEEE Trans. on Robotics and Automation, vol.8, no.5, 1992.

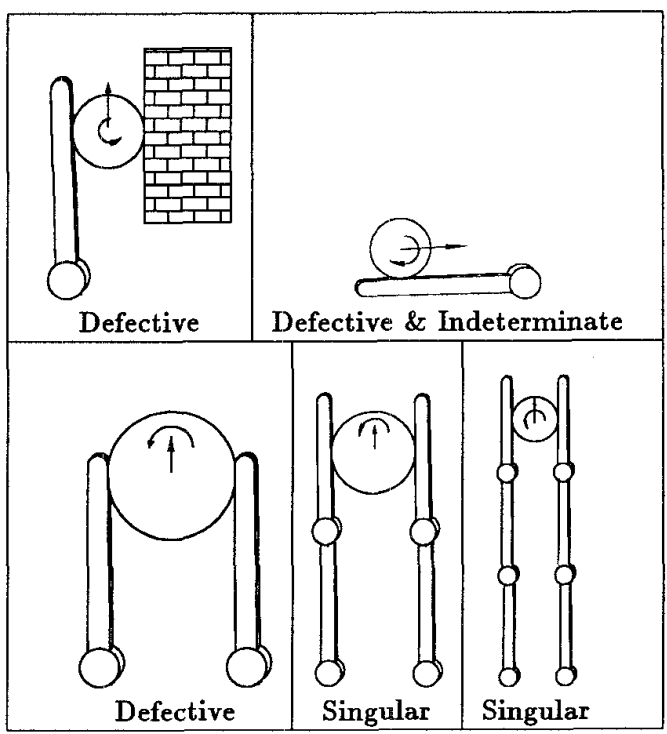

Table 2: Some uncontrollable modes for the examples of table 1.

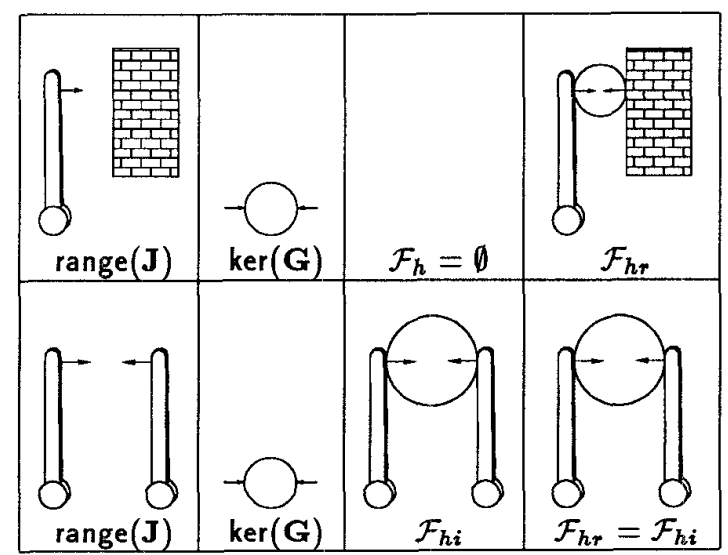

Table 3: Dynamically and steady-state (active) internal contact forces for the systems of examples 1 and 3 of table 1. Note that the existence of a nonvoid dynamically internal subspace for example 3 depends on the particular values assigned to geometric and inertial parameters. 